www.jmscr.igmpublication.org
Impact Factor 3.79

ISSN (e)-2347-176x

crossref DOI: http://dx.doi.org/10.18535/jmscr/v3i9.39

Journal Of Medical Science And Clinical Research

\title{
Role of Abdominal and Thoracic Ultrasonography in Dengue Fever
}

\author{
Authors \\ Dr Shilpa Hegde ${ }^{1 *}$, Dr Nita R Sutay ${ }^{2}$, Dr Mohammed Ashfaque Tinmaswala ${ }^{3}$ \\ ${ }^{1,3}$ Senior Resident, ${ }^{2}$ Professor and Head, Department of Pediatrics, Grant Government Medical College and \\ JJ Hospital Mumbai, Maharastra, India \\ *Corresponding Author \\ Dr Shilpa Hegde \\ Senior Resident \\ Department of Pediatrics, Grant Government Medical College and JJ Hospital Mumbai
}

\begin{abstract}
Aims and Objectives: 1) To Determine role of ultrasonography in early diagnosis of dengue fever.
\end{abstract}

2) To draw a co-relation between ultrasonography findings and the severity of dengue fever.

Study Design: A prospective study was conducted in Tertiary care centre in metropolitan during a period of 2 years.

Materials and Methods: All suspected cases of dengue based on clinical features and confirmed by laboratory profile between 2 months to 12 years were subjected to ultrasonography of abdomen \& thorax within $1^{\text {st }} 5$ days of fever spike. Dengue cases were classified according to the recent WHO classification 2011.

Results: Among the 100 cases studied it was found that dengue fever is common in male with a male to female ratio of 2.1:1.According to the Revised WHO dengue case classification 2011- 43 patients (43\%) had Nonsevere dengue without warning signs, 16 patients (16\%) had Non-severe dengue with warning signs \& 41 patients $(41 \%)$ had Severe dengue fever. In this study all the 100 patients were subjected to Ultrasonography of abdomen \& thorax within $1^{\text {st }} 5$ days of onset of fever or symptoms. Among them most common ultrasonography finding was Thickened gall bladder wall which was seen in 97 patients (97\%), hepatomegaly was present in 66 patients (66\%), Ascites in 55 patients (55\%), pleural effusion in 47 patients (47\%), Pericholecystic fluid in 25 patients $(25 \%)$ \& splenomegaly in 14 patients (14\%). Presence of pleural effusion \& as cites among the patients co-related with the severity of disease with $p<0.05$ showing significant association.

Conclusion: Abdominal \& thoracic ultrasonography in suspected dengue patients can be used in early diagnosis as it is easily available, non invasive \& cheap compared to serological studies. Findings can suggest the severity of the disease.

Key words: Dengue, Ultrasonography, Revised WHO classification.

\section{INTRODUCTION}

Dengue Fever is the most common arboviral disease in the world ${ }^{1}$.It is a mosquito-transmitted acute viral infection caused by 1 of 4 virus serotypes of the genus Flavivirus. Dengue fever (DF) has been known for more than a century in the tropical areas of South East Asia and the
Western Pacific regions ${ }^{2}$. A significant increase in the incidence of this infectious disease has taken place in the last 20years and Dengue has become a major international public health concern in recent years.

Dengue Fever Virus (DENV) is an RNA virus of the family Flaviviridae; genus Flavivirus. Dengue 
virus is transmitted by mosquitoes Aedes Aegypti, ${ }^{3}$.There are four strains of the virus, which are called serotypes, and these are referred to asDENV-1, DENV-2, DENV-3 and DENV-4 4 . All four serotypes can cause the full spectrum of disease $^{4}$. Infection with one serotype is believed to produce lifelong immunity to that serotype but only short term protection against the others ${ }^{5}$.

Incubation period is followed by three phases febrile, critical and Recovery. In Febrile phase patients develops high-grade fever suddenly classically described as biphasic or 'Saddle-back' pattern. This acute febrile phase usually lasts 2-7 days and is often accompanied by facial flushing, skin erythema, generalized body ache, myalgia, arthralgia, retro-orbital pain, photophobia, rubeliform exanthema and headache. Anorexia, nausea and vomiting are common.

Critical phase -In this phase patients with increased capillary permeability instead of improving may manifest with the warning signs, mostly as a result of plasma leakage. These are :Abdominal pain or tenderness, Persistent vomiting, Clinical fluid accumulation, Mucosalbleed, Lethargy; restlessness, Liver enlargement $>2 \mathrm{~cm}$. Laboratoryinvestigations may reveal increase in hematocrit concurrent with rapid decrease of platelet count.

Recovery phase -After 2-3 days, a gradual reabsorption of extravascular compartment fluid takes place in the following 48-72 hours.

Positive serology (anti dengue antibody) is the mainstay in the diagnosis of $\mathrm{DF}^{6}$. But serology takes approximately 7 days to give a positive result $^{7}$. The diagnosis of DF is often delayed owing to time taken for availability of serology test results. Moreover, this test is expensive and not widely available. Ultrasonography (USG) is a cheap, rapid and widely available non invasive imaging modality. In recent years several studies have concluded that ultrasonography of the chest and abdomen can be an important adjunct to clinical profile in diagnosis of DF and diagnosis can be made early in the course of the disease compared with other modes ofdiagnosis ${ }^{8}$. It can be used as a first-line imaging modality in patients with suspected DF to detect early signs suggestive of the disease prior to obtaining serologic confirmation test results, especially in a DF epidemic area 9.

Several literatures have described sonographic findings of DF. Some authors concluded that during an epidemic the ultrasound findings of gall bladder wall thickening with or without polyserositis in a febrile patient should suggest the possibility of $\mathrm{DF} / \mathrm{DHF}^{10}$. The reported changes vary according to the severity of each case. Studies have concluded that Ultrasound may be useful for early prediction of the severity of DHF in chidren ${ }^{11}$.

\section{Materials and Methods}

This prospective study was conducted in Tertiary care centre in metropolitan city over a period of 2 years. All suspected cases of dengue based on clinical features and confirmed by laboratory profile were subjected to Ultrasonography of abdomen \& thorax. All patients suspected of dengue fever based on clinical features and laboratory parameters were included in study between 2 months to 12 years of age. Cases were classified according to the Recent WHO classification of Dengue fever 2011.Dengue fever patients were clinically evaluated for vital signs \& systemic examination. Suspected children were investigated for complete blood count, Liver function tests, Chest $\mathrm{x}$ ray. All patients were serologically investigated with NS1 antigen, IgM, IgG dengue.

Tourniquet test (Hess capillary resistance test) was performed by placing the sphygmomanometer cuff around the upper arm and raising the pressure midway between systolic and diastolic pressure for 5minutes. The test was considered positive when more than 20 petechiae developed in an area of 1 sq. inch on the flexor aspect of forearm. Hemorrhagic manifestations usually appear 4-5 days after the onset of fever. This test is a very useful screening test in suspected cases of Dengue fever. 
All 100 cases were subjected to Ultrasonography of abdomen \& thorax within 5days of onset of fever or symptoms suggestive of dengue fever.

Ultrasonography of the abdomen was performed after 4-6hrs of fasting for better distention and visualisation of the gall bladder. In all the cases gall bladder was assessed for wall thickening as well as for pericholecystic fluid collection and for presence of calculi. Gall bladder wall thickness more than $3 \mathrm{~mm}$ was considered as gallbladder wall thickening. Splenic and liver size was measured along the long axis from dome to the tip $\&$ hepatorenal pouch and retro vesicular area in a supine position for ascites. Thoracic Ultrasonography was done in supine or sitting position with assessment of both the pleural spaces in the entire posterior and lateral thorax.

Table 1: WHO classification of Dengue fever 2011

\begin{tabular}{|c|c|c|}
\hline $\begin{array}{l}\text { Case Definition for Dengue } \\
\text { without warning signs }\end{array}$ & $\begin{array}{c}\text { Case Definition for } \\
\text { Dengue with warning signs }\end{array}$ & e Dengue \\
\hline 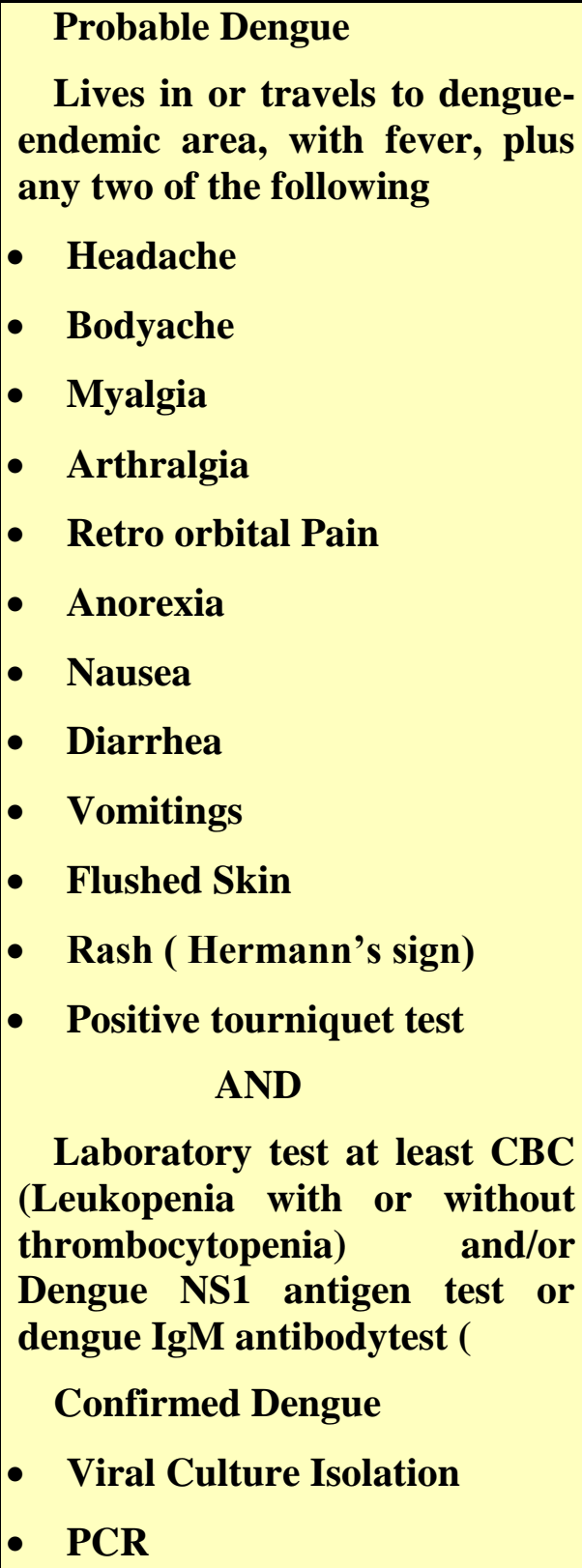 & $\begin{array}{l}\text { Probable Dengue } \\
\text { Lives in or travels to } \\
\text { dengue-endemic area with } \\
\text { fever lasting for 2-7 days } \\
\text { - Abdominal pain and } \\
\text { tenderness } \\
\text { - Persistent Vomitings } \\
\text { - Clinical signs of fluid } \\
\text { accumulation } \\
\text { - Mucosal bleeding } \\
\text { - Lethargy \& restlessness. } \\
\text { - Liver Enlargement } \\
\text { - Decreased or no urine } \\
\text { output within } 6 \text { hrs } \\
\text { - Laboratory } \\
\text { Increase in hematocrit or } \\
\text { decrease Platelet count }\end{array}$ & 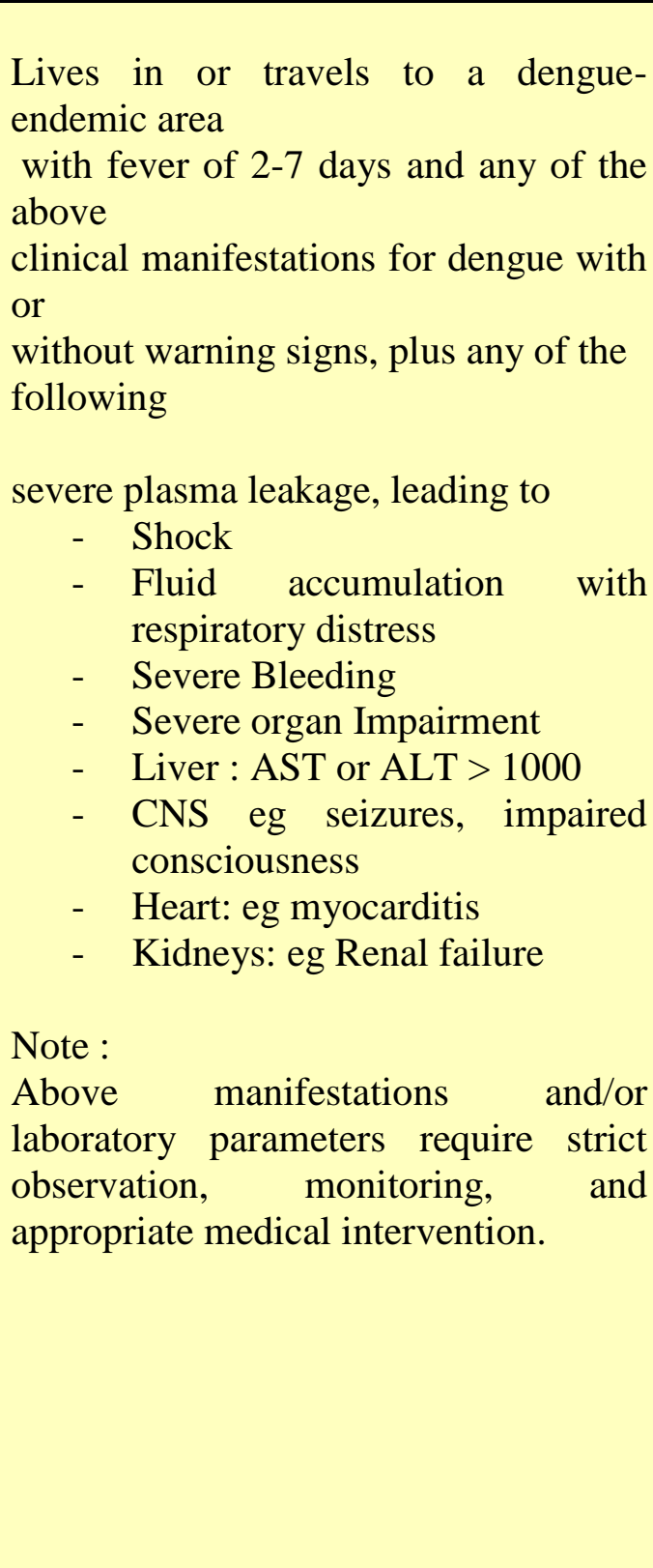 \\
\hline
\end{tabular}




\section{Results}

100 cases of dengue based on clinical and laboratory profile were subjected to Ultrasonography within 5days of onset of fever or clinical symptoms suggestive of dengue fever. Observations of these 100 cases were analysed.

According to the Revised WHO dengue case classification $^{12}$ 2011- 43 patients (43\%) had Nonsevere dengue without warning signs, 16 patients $(16 \%)$ had Non-severe dengue with warning signs \& 41 patients (41\%) had Severe dengue fever. 95cases $(95 \%)$ were cured \& $5(5 \%)$ died.

Among 100 cases studied $29 \%$ of cases were between the age group of 10-12years, $21 \%$ were between 6-8years, 16\% were between 4-6years, 14 $\%$ between 8 -10years, $11 \%$ between 2 -4years \& $9 \%$ in $2 \mathrm{mts}-2$ years children. $68 \%$ were male \& $32 \%$ were female. Males were more affected compared to females with the ratio of 2.1:1.

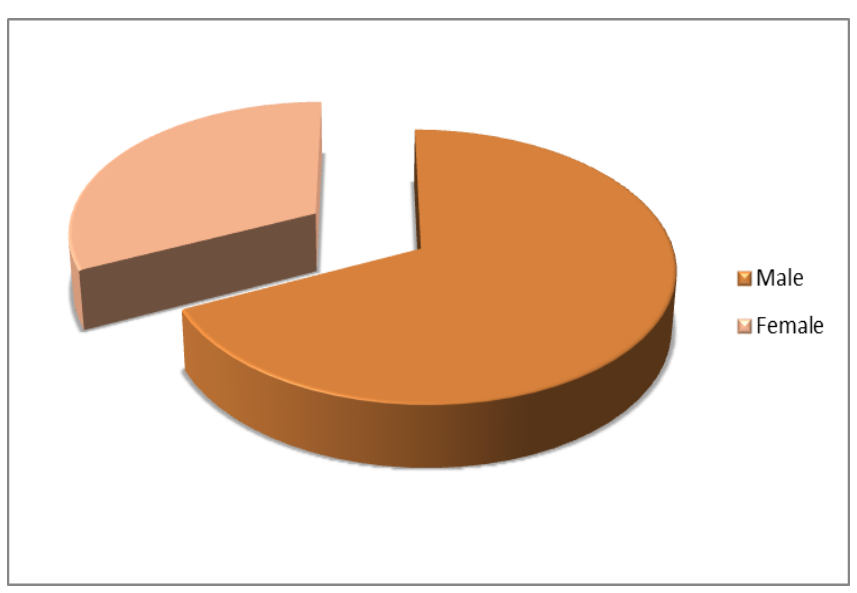

Fig 1: Gender distribution Amongst Cases

In this study $99 \%$ of patients had fever, which was the most common symptom found during the study. $92 \%$ patients had vomiting. Muskuloskeletal pain was present in $61 \%$ of patients. Skin rash was seen in $17 \%$ of the patients. Retro ocular pain was present in $33 \%$ of patients, congested eyes were present in $80 \%$ of patients, fever in $73 \%$, positive tourniquet test was seen in $62 \%$, petichae in $49 \%$, hypotension in $47 \%$, pallor in $33 \%$, rash in $32 \%$, tachypnea in $25 \%$, edema in $11 \%$, icterus in $6 \%$ \& lymphadenopathy in $1 \%$ of patients.
NS1 antigen was positive in $55 \%$, IgM was positive in $52 \%$ \& IgG was positive in $16 \%$ of the patients in our study. In this study Chest $X$ ray PA view was done in all admitted patients in which among $31 \%$ patients Chest $\mathrm{x}$ ray findings were suggestive of pleural effusion. $5 \%$ of the patients' chest $\mathrm{x}$ ray had bilateral fluffy infiltrates $\&$ maximum of $64 \%$ of patients Chest $x$ ray was within normal limits.

Table: 2. Ultrasonography findings in study population.

\begin{tabular}{|l|l|l|}
\hline USG findings & No. & $\begin{array}{l}\text { Percentage } \\
(\mathbf{n = 1 0 0})\end{array}$ \\
\hline Thickened gall bladder wall & 97 & $97.0 \%$ \\
\hline Hepatomegaly & 66 & $66.0 \%$ \\
\hline Pericholecystic fluid & 25 & $25.0 \%$ \\
\hline Splenomegaly & 14 & $14.0 \%$ \\
\hline Ascites & 55 & $55.0 \%$ \\
\hline Pleural effusion & 47 & $47.0 \%$ \\
\hline
\end{tabular}

In this study all the 100 patients were subjected to Ultrasonography of abdomen $\&$ thorax within $1^{\text {st }} 5$ days of onset of fever or symptoms. Among them most common finding was Thickened gall bladder wall which was seen in 97 patients (97\%). Next common finding was hepatomegaly which was present in 66 patients (66\%). Other findings like Ascites was present in 55 patients (55\%), pleural effusion was present in 47 patients $(47 \%)$, Pericholecystic fluid in 25 patients $(25 \%)$ \& splenomegaly in 14 patients $(14 \%)$.

The below graph shows association between ascites and severity of dengue fever In this study $23.3 \%$ of patients among Non severe without warning signs dengue patients had ascites on USG abdomen. $56.3 \%$ of patients among Non severe with warning signs had ascites \& $87.8 \%$ of the patients among severe dengue had ascites in USG finding. Statistical analysis of ascites shows $\mathrm{p}$ $0.211^{-8}$ which has significant association with the severity of the disease. $(p<0.01)$ 


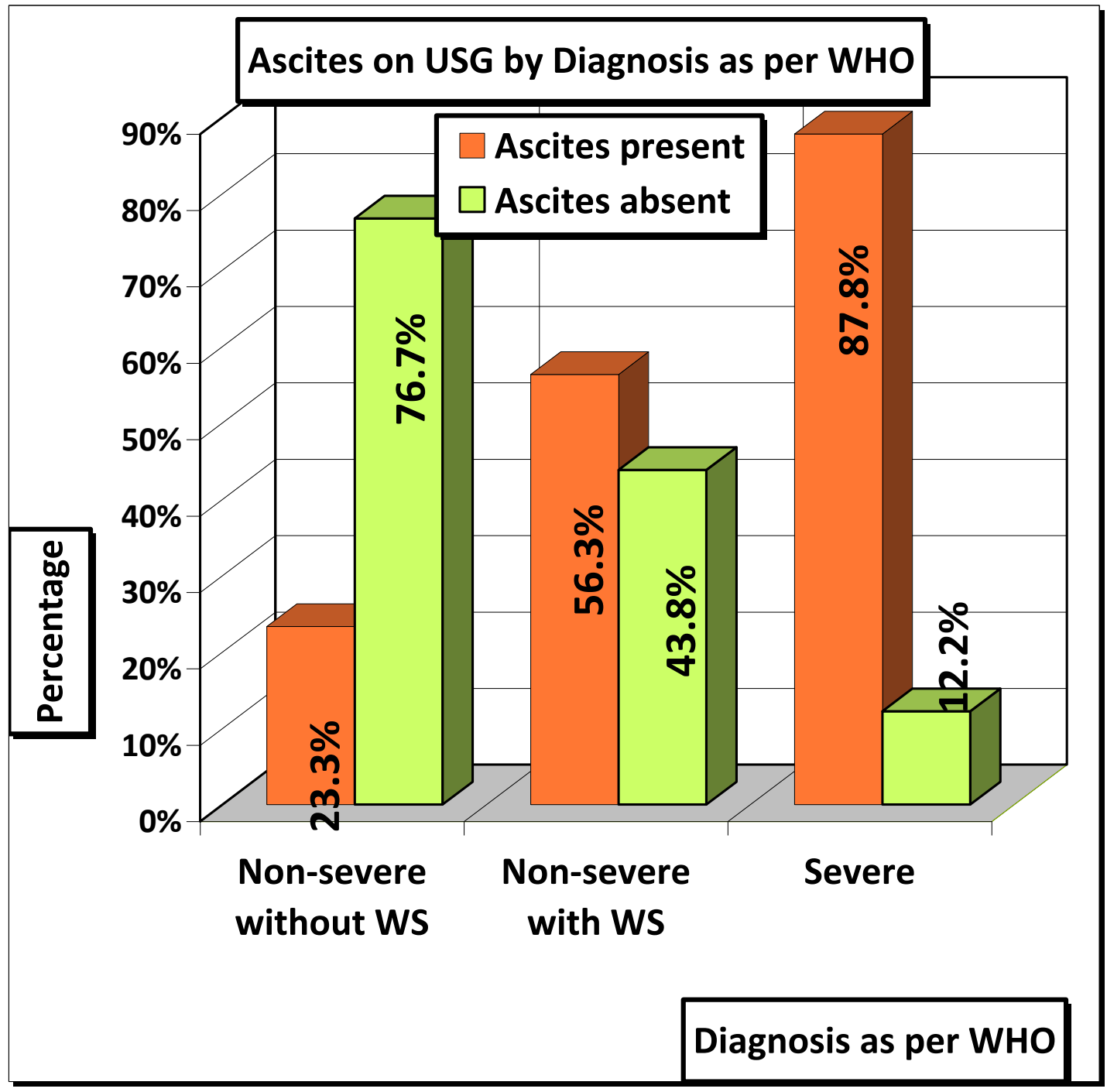

Fig: 2. Distribution of USG finding of Ascites in the study group.

The below graph shows association between pleural effusion and severity of dengue fever. In this study $4.7 \%$ of the patients among non severe without warning signs patients group showed presence of pleural effusion. 56.3\% patients of non severe with warning signs patients group showed presence of pleural effusion \& $87.8 \%$ of the patients among severe dengue had pleural effusion in USG finding. Statistical analysis of pleural effusion shows $\mathrm{p}-0.161^{-13}$ which has significant association with the severity of the disease. ( $p$ $<0.01)$. 


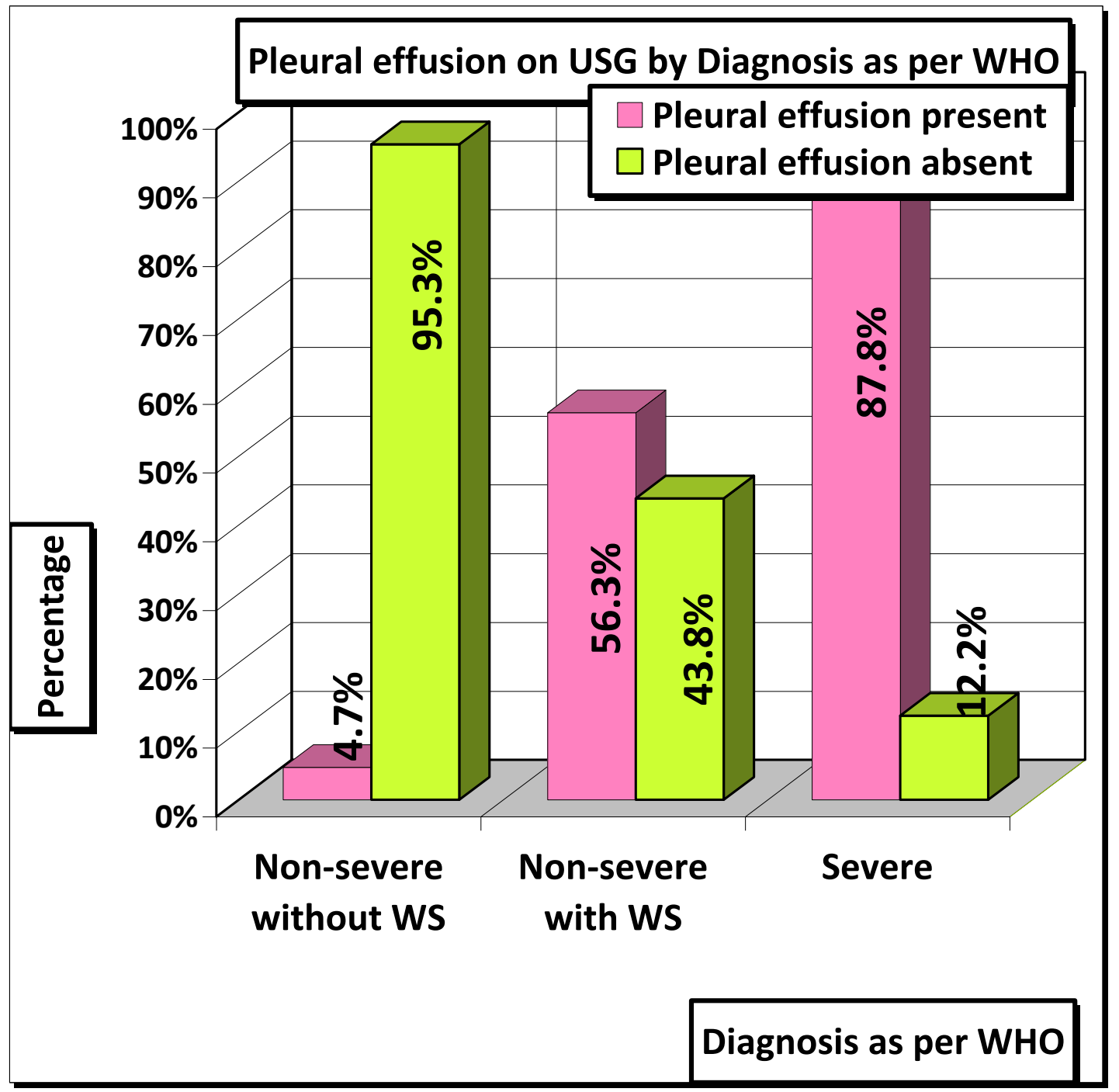

Fig: 3 : Distribution of USG finding of Pleural effusion in the study group

Most common age group of presentation in our study was 10-12 years, $29 \%$ of cases were between that age group with male to female ratio of $2.1: 1$.

The most common symptom found in this study was fever which was present in $99 \%$ of patients. Vomiting in 92\%, anorexia in $68 \%$, Muskuloskeletal pain in $61 \%$ of patients, generalised body ache in $59 \%$, abdominal pain in $36 \%$, restlessness in $17 \%$, Skin rash in $17 \%$, Retro ocular pain in $33 \%$ cases. Common findings observed among the cases were congested eyes in $80 \%$, petichae in $49 \%$, hypotension in $47 \%$, positive tourniquet test in $62 \%$, hepatomegaly in $42 \%$.

Out of 100 cases 43 patients (43\%) presented with dengue fever without warning signs, 16 patients
(16\%) presented with dengue fever with warning signs \& 41 patients $(41 \%)$ presented with severe dengue according to the revised WHO classification 2011. Out of 100 patients 95 patients $(95 \%)$ were cured \& 5 patients $(5 \%)$ were succumbed to death.

In our study all the 100 patients were subjected to Ultrasonography of abdomen \& thorax within $1^{\text {st }} 5$ days of onset of fever or symptoms. Among them most common finding was Thickened gall bladder wall which was seen in 97 patients (97\%). Next common finding was hepatomegaly which was present in 66 patients (66\%). Other findings like Ascites was present in 55 patients $(55 \%)$, pleural effusion was present in 47 patients (47\%), Pericholecystic fluid in 25 patients $(25 \%)$ \& splenomegaly in 14 patients $(14 \%)$. 
In this study $23.3 \%$ of patients among Non severe without warning signs dengue patients had ascites on USG abdomen. $56.3 \%$ of patients among Non severe with warning signs had ascites \& $87.8 \%$ of the patients among severe dengue had ascites in USG finding which has significant association with the severity of the disease. ( $p<0.01)$.

In this study $4.7 \%$ of the patients among non severe without warning signs patients group showed presence of pleural effusion. $56.3 \%$ patients of non severe with warning signs patients group showed presence of pleural effusion \& $87.8 \%$ of the patients among severe dengue had pleural effusion in USG finding which has significant association with the severity of the disease. $(p<0.01)$.

\section{Discussion}

The incidence of dengue fever has increased in India in recent past. Poor sanitation, water clogging and urbanisation are amongst one of important causes of breeding of mosquitoes which consequently is responsible for increase in mosquitoe borne diseases including dengue. Illiteracy and lack of awareness amongst general public about the breeding of mosquitoes and protection from their bites is important factors in determining vulnerability of people from getting infected with dengue ${ }^{13}$. Incidence of dengue feverincreases during the rainy season when breeding of vector mosquitoes is generally abundant. Dengue cases are more during September to November in the post-monsoon season $^{14}$.

Classical dengue fever clinically manifests with high fever with chills, the onset is sudden, severe headache, arthralgia and muscle pains, retroorbital pain and severe myalgia. Fever usually lasts for about 5 days and rarely for more than 7 days. Severe form of dengue fever is caused by simultaneous infection by more than 1 serotype because the first infection probably sensitizes the patient while the second infection with a different serotype appears to produce an immunological catastrophe ${ }^{15}$.Early detection of Dengue fever can go a long way in managing these patients and reducing morbidity and mortality, especially in severe dengue ${ }^{16,17}$.Early diagnosis of severe dengue can be aided by ultrasonography of abdomen and thorax. Balasubramanian $\mathrm{S}$ et al conducted study of 65 cases and concluded ultrasonography to be an ideal non-invasive investigation to detect plasma leakage and area specific hematocrit values to be useful as evidence of plasma leakage ${ }^{18}$. In a study performed by VenkataSai et al., ultrasonography was found to be higher in the later stages of the disease. Repeat ultrasonography on the fifth to seventh days detected pleural effusion in a significantly higher number of patients with dengue fever ${ }^{19}$.

The findings of our study were similar to studies conducted by Balasubramanian S etal, Venkata et al and Zaki S.A ${ }^{20}$.

\section{Conclusion}

Our study concludes that Thoracic \& abdominal sonography is a very important investigation in the diagnosis of dengue fever. Abdominal \& thoracic Sonography done within $1^{\text {st }} 5$ days of fever spike showing features of thickened gall bladder wall, pleural effusion, ascites, hepatomegaly and splenomegaly should strongly favor the diagnosis of dengue fever in patients presenting with fever and associated symptoms, particularly during an epidemic.Thoracic \&abdominal ultrasonography in clinically suspected cases of dengue is a good tool to aid in early diagnosis of dengue, even before the Dengue antibodies become detectable. This is useful especially in areas where Dengue NS1 testing is not available. Presence of pleural effusion \& ascites in thoracic \& abdominal ultrasonography respectively, increases with the severity of dengue fever as classified according to the new WHO classification. It is the cheap, non invasive \& easily available investigation compared to serology which is expensive.

Conflict of Interest- nil 


\section{References}

1. Keng-Liang $\mathrm{Wu}$, Chi-Sin Changchien, Chung-Huang Kuo, King-Wah Chiu, Sheng-Nan Lu, Chung-MouKuo, Yi-Chun Chiu, Yeh-Pin Chou. Early Abdominal Sonographic Findings In Patients With Dengue Fever. Journal ofclinical ultrasound October-2004,32:386-388.

2. Weekly epidemiological record. No. $6,8^{\text {th }}$ February 2002:41-3.

3. Gould EA, Solomon T (February 2008). "Pathogenic flaviviruses". Thelancet 371 (9611):500-9

4. H.J.Litz, H.A.Gharbi (2006). "Manual of diagnostic ultrasound in infectious Andtropical diseases"3.2.3 (89-92).

5. Chen LH, Wilson ME (October 2010). "Dengue and chikungunya infections intravelers".Curr.Opin. Infect. Dis. 23 (5): 438-44.

6. Rahman $M$, Rahman $K$, Siddique $A K$ et al.First outbreak of dengue haemorrhagic fever, Bangladesh. Emerg Infect Dis 2002; 8(7):738-40

7. Bhamarapravti N. Pathology of dengue haemorrhagic fever. In:Thongcharoen $\mathrm{P}$ (ed) Denge/dengue haemorrhagic fever. WHO, Geneva1993;pp.72-3

8. Fernanezlj. Dengue fever. In: lutzht \& Charbi ha (ed) Manual Of Diagnostic Ultrasound In Infectious Tropical Diseases, Springer-verlag, berlag, berlin 2006; pp.89-93.

9. $\mathrm{Wu} \mathrm{KL}$, Changchien $\mathrm{CS}$, Kuo $\mathrm{CH}$ etal. Early abdominal sonographic findings in patients with dengue fever. Clin Ultrasound 2004; 32(8): 386-8.

10. Sai PMV, Dev B, Krishnan R. Role of ultrasound in dengue fever. BritishJournal of Radiology 2005, 78:416-418.

11. Bharath Kumar Reddy KR, Laksmana RR, Veerappa BG, Shivananda. Ultrasonography as a tool in predicting the severity of dengue fever in children--a useful aid ina developing country. PediatrRadiol. 2013 Aug;43(8):971-7

12. Narvaez F, Gutierrez G, Pérez MA, et al. Evaluation of the Traditional and Revised WHO Classifications of Dengue Disease Severity. Hirayama K, ed.PLoS Neglected Tropical Diseases. 2011;5(11):e1397.

13. Singh B. Dengue outbreak in 2006: Failure of public health system? Indian J Community Med.2007;32:99-100.

14. Ukey P, Bondade S, Paunipagar P, Powar R, Akulwar S. Study of seroprevalence of dengue Fever in central India. Indian J Community Med. 2010;35:517-9.

15. Jawetz, Melwick .24th ed. McGraw Hill, Lange Publications; 2007.Adelberg's Medical Microbiology; pp. 350-5.

16. Lal M, Aggarwal A, Oberoi A. Dengue fever - An emerging viral fever in Ludhiana, North India. Indian J Public Health. 2007;51:198-9

17. Anders KL, Nguyet NM, Van VinhChau $\mathrm{N}$, et al. Epidemiological Factors Associated with Dengue Shock Syndrome and Mortality in Hospitalized Dengue Patients in Ho Chi Minh City, Vietnam. The American Journal of Tropical Medicine and Hygiene. 2011;84(1):127-134.

18. Balasubramanian S, Janakiraman L, Kumar SS, Muralinath S, Shivbalan S. A reappraisal of the criteria to diagnose plasma leakage in dengue hemorrhagic fever. Indian Pediatr. 2006;43:334-9.

19. VenkataSai PM, Dev B, Krishnan R. Role of ultrasound in dengue fever. $\mathrm{Br} \mathrm{J}$ Radiol. 2005;78:416-8.

20. Zaki SA. Pleural Effusion and Ultrasonography in Dengue Fever. Indian Journal of Community Medicine: Official Publication of Indian Association of Preventive \& Social Medicine. 2011;36(2):163. 